\title{
Vereinfachte Bewertung von Umweltbelastungen
}

\author{
Mensch und Umwelt sind vielfältigen Umwelt- \\ belastungen ausgesetzt. Mit integrierten \\ Methoden können auch Auswirkungen betrach- \\ tet werden, die in klassischen Ökobilanzen bis- \\ her nicht berücksichtigt wurden. \\ Von Christiane Markard, Michael Angrick, Markus \\ Berger, Matthias Finkbeiner, Jakob Frommer, \\ Ulrich Irmer, Klaus Müschen und Simone Richter
}

U $\mathrm{m}$ richtungsweisend Rat für die Einführung umweltfreundlicher Produkte oder die Bewertung verschiedener Technologien geben zu können, ist es oft erforderlich, unter verschiedenen Alternativen abzuwägen. Häufig stellt sich die Frage, ob bestimmte ökologische Verbesserungen wie die Energieersparnis nicht durch Verschlechterungen in einer oder mehreren anderen Belastungskategorien erkauft werden, die den vermeintlichen Vorteil wieder zunichtemachen.

Um in der täglichen Arbeit umweltbezogene Bewertungen oder Vergleiche vorzunehmen, sollte die gesamte Bandbreite der Belastungskategorien in den Blick genommen werden. $\mathrm{Zu}$ diesem Zweck hat das Umweltbundesamt (UBA) mit Unterstützung des Fachgebiets Sustainable Engineering der TU Berlin (Berger/Finkbeiner 2014) den Leitfaden „Vereinfachte Umweltbewertungen-VERUM“ entwickelt und an drei Fallstudien (Lampen, Handtrocknungssysteme, Gütertransporte) erprobt. Die Fallbeispiele (siehe Tabelle 2 und 3) sind in dem zugrunde liegenden Bericht ausführlich beschrieben. Durch die Veröffentlichung des VERUM-Leitfadens soll die UBA-interne Vorgehensweise der Öffentlichkeit transparent gemacht werden.

\section{Welche Belastungskategorien werden gebraucht?}

Wird über Umweltbelastungen diskutiert, so stehen zumeist die Treibhausgase als Verursacher des Klimawandels im Vordergrund. Auch Eutrophierung und Versauerung sind wichtige Größen, und schließlich werden noch die gefährlichen Stoffe genannt, die sich in der Umwelt ausbreiten und anreichern können. Viele lokal wirksame Umweltbelastungen haben durch die erfolgreiche Umweltpolitik der letzten Jahrzehnte in der öffentlichen Diskussion an Bedeutung verloren. Stattdessen stehen heute eher globale Probleme im Vordergrund, die in ihrem Ausmaß zum Teil bereits die Belastungsgrenzen des Pla- neten überschritten haben. Dazu gehören Klimaveränderung, Versauerung, stratosphärischer Ozonabbau, die Überlastung globaler Nährstoffkreisläufe, qualitative und quantitative Wasserübernutzung, Landnutzungsänderung, Biodiversitätsverlust, atmosphärische Aerosolbelastung und Chemikalienbelastung (Rockström et al. 2009). Für eine umfassende Bewertung müssen diese globalen Dimensionen durch weitere Kriterien für vor allem lokale Auswirkungen ergänzt werden.

Bei der Umweltbewertung von Technologien und Produkten muss die gesamte Bandbreite möglicher Belastungskategorien beurteilt werden. Für die vereinfachte Umweltbewertung war es daher zunächst erforderlich, alle potenziellen Belastungskategorien systematisch zu erfassen, um die Vollständigkeit des Katalogs der Belastungen sicherzustellen. Um ein umfassendes Bild über verschiedene mögliche Belastungen zu erhalten, hat das Umweltbundesamt Fallbeispiele aus den letzten zehn Jahren analysiert. Das Ergebnis sind fünf übergreifende Belastungsarten und 15 spezifische Belastungskategorien, die sich als so relevant erwiesen haben, dass sie generell bei einer Bewertung in Betracht zu ziehen sind (siehe Tabelle 1).

\section{Von der Ökobilanzmethode lernen}

Die vereinfachte Umweltbewertung wurde nicht als Alternative zur Ökobilanzmethode entwickelt, sondern als interne, integrierte Bewertungsmethode des Umweltbundesamtes. In einigen Punkten greift sie Elemente der Ökobilanz auf, in anderen Punkten wurden alternative Beurteilungsmöglichkeiten erarbeitet und zusätzliche Bewertungskategorien berücksichtigt.

\begin{tabular}{ll}
\hline Belastungsart & Belastungskategorie \\
\hline Chemische Belastungen & Treibhausgase \\
& Schadstoffe/Nährstoffe in Außenluft \\
& Schadstoffe in Innenräumen \\
& Abwasser \\
& Diffuse Nähr- und Schadstoffeinträge, Pestizide \\
\hline Physikalische Belastungen & Lärm \\
& Strahlung \\
& Mechanische Tötung von Tieren \\
\hline Biologische Belastungen & Krankheitserreger \\
& Invasoren \\
\hline Ressourcen- & Verbrauch mineralischer Rohstoffe inklusive \\
inanspruchnahme & fossiler Energieträger \\
& Verbrauch biotischer Rohstoffe \\
& Wasserverbrauch \\
\hline Regelwidriger Umgang & Naturraumbeanspruchung \\
\hline
\end{tabular}

Tabelle 1: Belastungsarten und -kategorien in VERUM 


\begin{tabular}{|c|c|c|}
\hline Belastungsart & Belastungskategorie & Reihenfolge der zu untersuchenden Alternativen \\
\hline \multirow[t]{3}{*}{ Chemische Belastungen } & Treibhausgase & 1. Kompaktleuchtstofflampe, 2. Glühlampe \\
\hline & Gefährliche Stoffe Außenluft & gleichwertig \\
\hline & Stoffbelastung im Innenraum & gleichwertig (keine Gefährdung) \\
\hline Physikalische Belastungen & Strahlung & $\begin{array}{l}\text { 1. Glühlampe, 2. Kompaktleuchtstofflampe } \\
\text { (jedoch in beiden Fällen keine Gefährdung) }\end{array}$ \\
\hline Ressourcen & Verbrauch mineralischer Rohstoffe und fossiler Energieträger & 1. Glühlampe, 2. Kompaktleuchtstofflampe \\
\hline Störfälle/Unfälle & Quecksilberemission bei Lampenbruch & $\begin{array}{l}\text { 1. Glühlampe, 2. Kompaktleuchtstofflampe } \\
\text { (jedoch keine Gefährdung) }\end{array}$ \\
\hline
\end{tabular}

Tabelle 2: Bewertung Glühlampe versus Kompaktleuchtstofflampe auf Produktebene (Oehme et al. 2013)

\begin{tabular}{|c|c|c|}
\hline Belastungskategorie & Belastung durch Produktgesamtheit Glühlampe & $\begin{array}{l}\text { Belastung durch Produktgesamtheit } \\
\text { Kompaktleuchtstofflampe }\end{array}$ \\
\hline Treibhausgase & $\begin{array}{l}\text { nicht tolerierbar (>ca. } 75-80 \% \text { als Kompaktleuchtstoff- } \\
\text { lampe oder LED-Lampe, Einstufung als nicht tolerierbar } \\
\text { bereits ab }>30 \% \text { zur Referenz) }\end{array}$ & $\begin{array}{l}\text { geringe bis mäßige Belastung } \\
\text { (gleich bis }>10 \% \text { gegenüber LED-Lampe) }\end{array}$ \\
\hline $\begin{array}{l}\text { Gefährliche Stoffe Außenluft } \\
\text { (Quecksilberbilanz) }\end{array}$ & gleichwertig & gleichwertig \\
\hline Stoffbelastung im Innenraum & geringe Belastung & geringe Belastung \\
\hline Strahlung & Belastung unterhalb Grenzwert & Belastung unterhalb Grenzwert \\
\hline $\begin{array}{l}\text { Quecksilberemission } \\
\text { bei Lampenbruch }\end{array}$ & keine Belastung & $\begin{array}{l}\text { Schwere der gesundheitlichen Beeinträchtigung: gering } \\
\text { Anzahl der betroffenen Personen: gering } \\
\text { Eintrittswahrscheinlichkeit: hoch }\end{array}$ \\
\hline
\end{tabular}

Tabelle 3: Bewertung der Gesamtbelastung Glühlampe versus Kompaktleuchtstofflampe (Oehme et al. 2013)

Es liegt nahe, technische Neuentwicklungen zunächst nur hinsichtlich ihrer Nutzungsphase zu beurteilen. Der Konsument kann die Umweltfolgen, wenn überhaupt, oft nur für den Zeitraum, in dem er das Produkt besitzt und nutzt, beurteilen. Entscheidet er sich zum Beispiel für ein energiesparsames Produkt, so will und kann er nicht auch noch prüfen, ob dies im Herstellungsprozess zu kritischen Belastungen durch die Nutzung seltener Rohstoffe oder durch Emissionen führt. „Von der Wiege bis zur Bahre" ist daher eine unabdingbare Prämisse, die die Ökobilanzmethode (ISO 2006a, 2006b) vorgibt, um solche Fehlbeurteilungen zu vermeiden. Diese übergreifende Sichtweise wurde für die Vereinfachte Umweltbewertung übernommen. Übertragbar ist auch die Normierung der Bewertung auf die sogenannte "funktionelle Einheit", um für einen bestimmten Nutzen, der durch verschiedene Technologien (Glühlampe, Leuchtdiode, Kompaktleuchtstofflampe oder Kerze) realisiert werden kann, eine Vergleichbarkeit herzustellen. Schließlich wurde im Rahmen der Ökobilanzmethodik eine Reihe von Instrumenten entwickelt, insbesondere Datenbanken für Sachbilanz - Inventare verschiedener Softwareanbieter, die auch bei anderen Vorgehensweisen hilfreich sind, um die notwendige Datenbasis zu beschaffen (Umweltbundesamt o. J., PE International o. J., Ecoinvent o. J.).

\section{Vereinfachtes Vorgehen}

Die vollständige Liste der Belastungskategorien ist ein Hilfsmittel, um keine der vielfältigen Umweltbelastungen zu übersehen. Sie hilft in der Anwendung aber auch, die relevanten
Umweltbelastungen von den vernachlässigbaren zu unterscheiden und die Liste der Prüfkriterien einzuengen. Im ersten Schritt der Vereinfachten Umweltbewertung wird geprüft, welche Belastungskategorien für die anstehende Bewertung von Bedeutung sind. Dabei scheiden Kriterien aus, die nicht nennenswert zu einer Umweltbelastung beitragen. Geht es also um ein Produkt, das nicht mit Energie betrieben wird, so sind Treibhausgase in der Regel nicht zu beurteilen. So kann die Zahl der zu betrachtenden Belastungskategorien sukzessive eingeengt werden. Führt eine technische Neuentwicklung hingegen dazu, dass rechtlich verbindlichen Umweltziele nicht eingehalten werden (zum Beispiel Autoklimaanlagen mit fluorierten Kältemitteln), wird die Umweltbewertung an diesem Punkt mit dem Ergebnis beendet, dass das Produkt grundsätzlich nicht als umweltfreundlich eingeschätzt werden kann (Hoffmann/Plehn 2010).

\section{VERUM als Risikoscreening-Tool}

VERUM erweist sich auch als geeignetes Risikoscreening-Tool. Die Beurteilung neuer Entwicklungen im Energiebereich stellte das Umweltbundesamt vor ganz neue Fragen. $\mathrm{Zu}$ Beginn der Diskussion über die Möglichkeiten der Kohlenstoffdioxid-Verpressung in den Untergrund (CCS - Carbon Capture and Storage) wie auch über die unkonventionelle Gasförderung durch Fracking waren die Bewertungskriterien völlig offen (Becker et al. 2009, Kirschbaum 2012). Beide Techniken waren für das UBA neu und hinsichtlich ihrer potenziellen Auswirkungen auf Mensch und Umwelt zunächst schwer fass- 


\begin{tabular}{|c|c|c|c|c|c|}
\hline Indikator & keine Belastung & geringe Belastung & mäßige Belastung & hohe Belastung & nicht tolerierbare Belastung \\
\hline $\begin{array}{l}\text { Experten- } \\
\text { einschät- } \\
\text { zung }\end{array}$ & $\begin{array}{l}\text { Bewertungsvoraussetzun- } \\
\text { gen aus Vorprüfung nicht } \\
\text { erfüllt, d. h. es werden } \\
\text { keine Nähr- oder Schad- } \\
\text { stoffe freigesetzt. }\end{array}$ & $\begin{array}{l}\text { Geringe Stofffreisetzung, } \\
\text { d. h. geringes Risiko, UQN } \\
\text { zu überschreiten - in der } \\
\text { Regel bewegen sich die } \\
\text { Konzentrationen im Be- } \\
\text { reich < } 1 / 2 \text { UQN (nur sehr } \\
\text { lokale Überschreitungen). }\end{array}$ & $\begin{array}{l}\text { Erhöhung der Belastung, } \\
\text { UQN langfristig nicht } \\
\text { gefährdet, d. h. mäßiges Ri- } \\
\text { siko, UQN zu überschrei- } \\
\text { ten. In der Regel bewegen } \\
\text { sich die Konzentrationen } \\
\text { im Bereich < UQN. Risiko } \\
\text { steigender Trends (eher } \\
\text { lokal und regional begrenz- } \\
\text { te Überschreitungen). }\end{array}$ & $\begin{array}{l}\text { Erhöhung der Belastung, } \\
\text { UQN langfristig gefährdet, } \\
\text { Gefährdung von Pflanzen } \\
\text { und Tieren, d. h. hohes Risiko, } \\
\text { UQN zu überschreiten. An } \\
\text { bis zu 10\% der Messstellen } \\
\text { werden die UQN nicht einge- } \\
\text { halten. Sehr hohes Risiko für } \\
\text { steigende Nähr- und Schad- } \\
\text { stofftrends (regional größere } \\
\text { Zahl von Überschreitungen } \\
\text { zu erwarten). }\end{array}$ & $\begin{array}{l}\text { Erhöhung der Belastung } \\
\text { führt zu Überschreitung } \\
\text { des UQN, Gesundheitsge- } \\
\text { fährdung, d. h. UQN wer- } \\
\text { den mit großer Wahrschein- } \\
\text { lichkeit überschritten. In } \\
\text { der Regel bewegen sich die } \\
\text { Konzentrationen im Be- } \\
\text { reich > UQN (Überregio- } \\
\text { nale Überschreitungen sind } \\
\text { zu erwarten). }\end{array}$ \\
\hline
\end{tabular}

Tabelle 4: Bewertungsmodell der Belastungskategorie diffuse Nährstoff- und Schadstoffeinträge in Wasser (UQN: Umweltqualitätsnorm)

bar. Es fehlten Prüfkriterien, nach denen systematisch vorgegangen werden konnte und die jeweiligen Vor- und Nachteile der Techniken, sofern es auch um Konkurrenzen hinsichtlich der unterirdischen Raumplanung oder der Forschungs- und Investitionsressourcen ging, abgewogen werden konnten.

Das Umweltbundesamt hat daher die Belastungskategorien von VERUM zunächst genutzt, um ein Risikoscreening vorzunehmen. Damit konnte deutlich gemacht werden, welche Vorund Nachteile die Technologien haben, und welche Kategorien einer vertieften Prüfung bedürfen.

Zweifelsohne war die tabellarische Aufbereitung für eine abschließende Bewertung dieser Technologien nicht ausreichend. Hilfreich war sie jedoch für das weitere Vorgehen und als Grundlage, um Schwerpunkte des Forschungsbedarfs zu identifizieren.

\section{Vom einzelnen Produkt zur Produktgesamtheit}

VERUM sieht vor, dass die Bewertung in zwei Schritten erfolgt. Zunächst erfolgt der Vergleich auf der Ebene der funktionellen Einheit, also auf der Produktebene. Sie ermöglicht eine Priorisierung der zu untersuchenden Alternativen innerhalb einer Belastungskategorie. Die Rangbildung erfolgt auf Basis der jeweiligen Bewertungsmodelle der Belastungskategorien (siehe Tabelle 4).

Da jedoch auch geringe spezifische Belastungen relevante Konsequenzen haben können, wenn das Produkt in hohen Stückzahlen produziert wird, ist im zweiten Schritt auch eine Bewertung der durch die Produktgesamtheit hervorgerufenen Belastungen erforderlich. Dieses Faktum hat zwar keine Auswirkungen auf den Vergleich von Alternativen, da dieser über die funktionelle Einheit gegeben ist, wohl aber auf die Belastungsbewertung des Produktes insgesamt.

\section{Eichung oder Interkalibrierung von Bewertungskriterien}

Im zweiten Schritt werden die als relevant erkannten Belastungskategorien quantitativ oder qualitativ bewertet. Ziel der vereinfachten Umweltbewertung (VERUM) ist es, auch ohne vollständige quantitative Untersuchungen relativ schnell zu einer ersten plausiblen Gesamtbewertung zu kommen. VERUM beruht auf den zwei Grundsätzen „so gut wie möglich“, aber auch „Anwendbarkeit vor Genauigkeit“. Das heißt, die zu untersuchenden Alternativen sollen möglichst umfangreich und auf der bestmöglichen Datenbasis bewertet werden. Doch auch bei unvollständiger Datenlage soll die Methode anwendbar bleiben, indem Vereinfachungen und qualitative Abschätzungen zulässig sind. Allerdings muss sichergestellt werden, dass die Unzulänglichkeiten hinsichtlich Datengenauigkeit und -vollständigkeit transparent dargestellt und in der Interpretation der Ergebnisse berücksichtigt werden.

Eine Aggregation der Bewertung aller Wirkungskategorien zu einem Wert wird mit VERUM nicht angestrebt. Es wurde vielmehr versucht, für alle Wirkungskategorien fünf Belastungsklassen konsistent und einheitlich zu definieren: „keine“, "geringe“, „mäßige“, „hohe“ oder „nicht tolerierbare Belastung“. Vorbild war das fünfklassige Bewertungsschema der EU-Wasserrahmenrichtlinie, das den „sehr guten“, „guten“ „mäßigen“, „unbefriedigenden“ und „schlechten Gewässerzustand“ für die Einstufung vorsieht. Zum Zweck der Klassifizierung wurden Bewertungsmodelle und -kriterien für jede Belastungsklasse beschrieben und soweit möglich mit Zahlenwerten unterlegt. Sie ermöglichen die quantitative oder qualitative Abschätzung, ob bei einer Produkt- oder Technikeinführung die Produktgesamtheit mehr oder minder hohe Beiträge zu Umwelt- und Gesundheitsbelastung leistet.

Für viele Belastungskategorien sind sogenannte Umweltqualitätsziele abgeleitet. Umweltqualitätsziele gibt es für nahezu alle chemischen Stoffe in Wasser, Boden, Luft und Innenräumen, Strahlung sowie für Lärm, zum Teil auch für mikrobielle Belastungen. Für diese Belastungskategorien erfolgt die „Interkalibrierung“ durch das Qualitätsziel. Im Fall der Belastungskategorie „Diffuse Nähr- und Schadstoffeinträge in Wasser“ liegt eine „mäßige Belastung“ vor, wenn das Qualitätsziel erreicht, aber nicht langfristig überschritten wird. Als „gering“ ist eine Belastung anzusehen, die sich im Bereich des halben Qualitätsziels bewegt. Bei einer „hohen Belastung“ wird das Qualitätsziel häufiger oder regional in größerer Zahl über- 
schritten. Die Kategorien „keine“ oder „nicht tolerierbare Belastung" sind in der Regel für einen konkreten Vergleich nicht von Bedeutung, da die Kategorien entweder aufgrund fehlender Relevanz nicht betrachtet werden können oder das Produkt aufgrund nicht eingehaltener Anforderungen grundsätzlich nicht empfehlenswert ist. Umweltqualitätsziele sind in Rechtsvorschriften und Publikationen relevanter Organisationen veröffentlicht. $\mathrm{Zu}$ diesen Publikationen gehören zum Beispiel die Oberflächengewässerverordnung 2011 oder die Air Quality Guidelines der WHO (Kryzanowski/Cohen 2008).

Methodisch schwieriger ist die Aufstellung eines Klassifikationsschemas für die Belastungsschwere bei Kategorien, für die keine Umweltqualitätsziele bestehen oder ableitbar sind. Daher wurden für Treibhausgasemissionen oder Flächenverbrauch hilfsweise die sogenannten Nachhaltigkeitsziele der Bundesregierung herangezogen, die bis 2020 erreicht werden sollen (Bundesregierung 2002). Einzustufen ist hier, inwieweit die zu beurteilenden Produkte und Techniken diesen Zielen förderlich sind oder ihnen entgegenstehen. Für den Klimaschutz gilt das 40-Prozent-Ziel bis 2020 im Vergleich zu 1990. Bei der Flächeninanspruchnahme durch Siedlungen und Verkehr oder Bergbau wird die Erreichbarkeit des 30-Hektar-Ziels herangezogen, ergänzt durch den Grad der zumeist irreversiblen Flächennutzungsänderung. Bei terrestrischen Flächen beurteilt man den Grad der Verschlechterung der relevanten Nutzungstypen (von „naturnah" bis „degradiert“), bei Gewässern die Verschlechterung der Strukturgüte (von „unverändert“ bis „vollständig verändert").

Die Beurteilung der mechanischen Tötung von Tieren oder der Ausbreitung invasiver Arten ist unter anderem bei technischen Bauwerken relevant, die wandernde Arten behindern oder unerwünscht fördern. Dabei werden die Kriterien für den Schutz der Biodiversität nicht auf das Individuum, sondern auf den Erhalt der Population fokussiert. Bewertet wird daher, ob die Auswirkungen temporär und regional sind oder ob sie, zum Beispiel bei bestimmten Wasserkraftanlagen, dauerhaft den Erhalt der Population gefährden. Insofern besteht hier auch Kongruenz mit der ökotoxikologischen Bewertung gefährlicher Stoffe, die sich auch auf die Gefährdung der Lebensgemeinschaften bezieht.

Das Risiko der Ausbreitung von Krankheitserregern zählt zu den Belastungskategorien, die vor allem bei energiesparenden Techniken und Produkten und bei Recyclingprozessen zu berücksichtigen sind. So kann beispielsweise die Absenkung von Bereitstellungstemperaturen in Wasserboilern zwar den Energieverbrauch senken, dafür aber die Ausbreitung von Legionellen fördern. Methodisch erfolgt die Risikoabschätzung durch Krankheitserreger durch eine qualitative/semiquantitative Experteneinschätzung. Die relevanten Kriterien sind die Schwere der Erkrankung und die Erkrankungswahrscheinlichkeit, um das durch einen Erreger hervorgerufene Gesundheitsrisiko als gering, mittel oder hoch zu bewerten.

Für die Umweltbelastung durch Ressourceninanspruchnahme existiert derzeit noch kein verallgemeinerbarer Bewer- tungsansatz, zumal die Unterschiede zwischen regenerierbaren (Wasser, biologische Ressourcen) und nicht regenerierbaren Ressourcen (einschließlich Energieträger und Böden) ein unterschiedliches Herangehen erfordern.

Bei mineralischen Rohstoffen spielen verschiedene Kriterien eine Rolle. Besonders relevant sind das Versorgungsrisiko und die Möglichkeit der Wiedergewinnung am Ende des Produktlebenszyklus. Hilfreich ist der sogenannte Kritikalitätsindex für Deutschland (Erdmann et al. 2011), der für die jeweiligen Rohstoffe eine Experteneinschätzung erlaubt. Bei der Beurteilung des Verbrauchs an fossilen Energieträgern erfolgt eine Experteneinschätzung, ob die Produktgesamtheit die Erreichung des Primärenergieverbrauchsziels der nationalen Nachhaltigkeitsstrategie (Senkung um 20\% bis 2020 gegenüber 2008) gefährdet.

Im Unterschied zu nicht regenerativen Ressourcen sind biotische Rohstoffe zumindest theoretisch unbegrenzt vorhanden und werden lediglich durch das Vorhandensein anderer Ressourcen (insbesondere Fläche und Wasser) begrenzt. Allerdings kann bei Übernutzung und Nutzungskonkurrenzen der Einsatz biotischer Rohstoffe ein reales Problem sein (zum Beispiel Überfischung; Knappheit von Ackerflächen, die in globalem Maßstab zu Entwaldung führt), das zu adressieren ist. Das Maß für die Knappheit wird zum Beispiel bei Fischerei durch das Verhältnis von Entnahme- zu Erneuerungsraten bestimmt, das heißt dadurch, wie weit die jährliche Entnahmerate der biotischen Ressourcen die Erneuerungsrate über- oder unterschreitet. Angesichts knapper fruchtbarer Böden kann beim Produktvergleich auch der jeweilige Flächenbedarf an landund forstwirtschaftlichen Flächen für die Erzeugung von Biomasse herangezogen werden.

Auch für die Bewertung der Auswirkungen des Wasserverbrauchs sind Informationen zum Ort des Wasserverbrauchs und der dort herrschenden Wasserknappheit erforderlich. Je nach Datenlage erfolgt die Bewertung entweder über die lokale Wasserknappheit, unabhängig vom verbrauchten Volumen. Kriterium für diese Bewertung ist der lokale Wasserstressindex nach Pfister et al. (2009). Liegen regionale quantitative Daten zum produktspezifischen Wasserverbrauch entlang des Produktlebensweges vor, werden die lokalen Verbräuche mithilfe des lokalen Wasserstressindexes gewichtet. Bei der Beurteilung der Gesamtbelastung durch ein Produkt oder eine Technik wird abgeschätzt, ob infolge des dadurch induzierten Wasserverbrauchs eine regionale Verschärfung der Wasserknappheit zu erwarten ist.

Beim Einsatz und Betrieb von Produkten und Techniken geht man normalerweise vom ordnungsgemäßen Betrieb aus. Der wird auch bei einer Beurteilung nach VERUM zugrunde gelegt. Dennoch kann bei einem Vergleich verschiedener Techniken (zum Beispiel Atomkraftwerk versus Kohlekraftwerk) die inhärente Störfallgeneigtheit einen wesentlichen Bewertungsunterschied machen. Das Risiko ist definiert durch Eintrittswahrscheinlichkeit und Schwere des Schadens sowie seine Behebbarkeit. 


\section{Wie vergleicht man unterschiedliche Belastungen?}

Möchte man verschiedene Umweltbelastungen miteinander vergleichen, so stellt sich die Frage der Umrechnung. Das mag innerhalb einer Kategorie noch einfach erscheinen, aber wie vergleicht man eine Lärmbelastung mit einem erhöhten Erkrankungsrisiko durch Krankheitserreger? Ist eine „geringe Lärmbelastung“ mit einer "geringen mikrobiellen Belastung“ vergleichbar? Für eine größtmögliche Transparenz wird bei VERUM zunächst keine Gesamtbewertung angestrebt, sondern es werden die Ergebnisse der Beurteilung in den Belastungsarten oder Belastungskategorien getrennt dargestellt. Damit können für unterschiedliche Anwendungsbereiche bei der Bewertung unterschiedliche Schwerpunkte gesetzt werden. Für die Anwendung im Krankenhaus kann zum Beispiel ein Produkt gewählt werden, bei dem das Infektionsrisiko durch Krankheitserreger besonders gering ist, während in weniger kritischen Bereichen dem Alternativprodukt mit der besseren Energiebilanz der Vorzug gegeben werden kann.

Die Beurteilung komplexer Sachverhalte mit einfacher oder vereinfachter Methodik erfordert in besonderem Maße Vorsichtsmaßnahmen, um Fehlschlüsse zu vermeiden. Für das Umweltbundesamt ist es wichtig, die Aussagekraft von mit vereinfachter Umweltbewertung nach VERUM erzielten Ergebnissen einzuschätzen. Da eine quantitative Unsicherheitsermittlung aufgrund unzureichender Daten und des oft qualitativen Charakters der Bewertung nicht möglich ist, muss die Aussagesicherheit ebenfalls qualitativ beurteilt werden. Hierzu wurden Kriterien entwickelt.

Je nach Fragestellung und Zweck kann die erforderliche Belastbarkeit des Ergebnisses unterschiedlich sein. Umweltpolitische Entscheidungen, die weitreichende rechtliche oder ökonomische Konsequenzen haben, werden jedoch immer eine solide Datenbasis erforderlich machen.

\section{Schlussfolgerung}

In der täglichen Arbeit des Umweltbundesamtes ist eine Bewertung nach VERUM für viele Fragestellungen hinreichend richtungsweisend und für die Zwecke der ersten Beratung ausreichend. Auf jeden Fall ermöglicht sie ein Pre-Screening, um $\mathrm{zu}$ ermitteln, welches die relevanten Belastungskategorien sind, ob die Datenlage für eine vereinfachte Umweltbewertung ausreicht und für welche Belastungskategorien weitere Daten zu erheben sind. Ein detaillierterer Vergleich mit einer international anerkannten Methode ist insbesondere dann erforderlich, wenn es sich um eine umweltpolitisch relevante Fragestellung handelt.

Das Umweltbundesamt ist eine Institution von Expertinnen und Experten. Für alle relevanten Belastungskategorien und Umweltwirkungen wirken Fachspezialisten bei der Entwicklung von Bewertungsmethoden auf nationaler und internationaler Ebene mit. Dabei ist es nicht immer trivial, im Zusam- menspiel der Methoden die Vergleichbarkeit und die Kohärenz zu wahren. Eine große Institution wie das Umweltbundesamt muss jedoch sicherstellen, dass nicht von Fall zu Fall mit unterschiedlichem Maßstab gearbeitet wird. Auch diesem Zweck dient VERUM.

\section{Literatur}

Becker, R. et al. (2009): CCS - Environmental protection framework for an emerging technology. Hintergrundpapier, Umweltbundesamt.

Berger, M./Finkbeiner, M. (2014): Vereinfachte Umweltbewertungen des Umweltbundesamtes (VERUM). UBA-TEXTE 33/2014.

Bundesregierung (Hrsg.) (2002): Perspektiven für Deutschland - Unsere Strategie für eine nachhaltige Entwicklung. Berlin.

Ecoinvent centre (o. .). : http://www.ecoinvent.org/. Aufgerufen 10.06. 2012.

Erdmann, L. et al. (2011): Kritische Rohstoffe für Deutschland. Institut für Zukunftsstudien und Technologiebewertung (IZT) und Adelphi, Berlin. Gutachten im Auftrag der KfW Bankengruppe.

Hoffmann, G./Plehn, W. (2010): Natürliche Kältemittel in PKW-Klimaanlagen ein Beitrag zum Klimaschutz. Hintergrundpapier, Umweltbundesamt.

ISO (2006a): Environmental management - Life cycle assessment Principles and framework (ISO 14.040:2006), International Organisation for Standardisation. Genf.

ISO (2006b): Environmental management - Life cycle assessment - Requirements and guidelines (ISO 14.044:2006), International Organisation for Standardisation. Genf.

Kirschbaum, B. (2012): Gewinnung von Erdgas aus unkonventionellen Lagerstätten - Auswirkungen auf die Umwelt. UMID Heft 1. S. 30-35.

Krzyzanowski, M./Cohen, A. (2008): Update of WHO air quality guidelines. Air Qual Atmos Health 1. S.7-13.

Oberflächengewässerverordnung vom 20. Juli 2011, BGBI. I S. 1429.

Oehme, I. et al. (2013): Fallbeispiel Lampen - Clühlampe versus Kompaktleuchtstofflampe. In: Berger a. a. O.

PE International (o. .). : http://www.gabi-software.com. Aufgerufen 12.06. 2013.

Pfister, S. et al. (2009): Assessing the environmental impacts of freshwater consumption in LCA. Environmental Science \& Technology 43. S. 4.098-4.104.

Rockström, J. et al. (2009): Planetary Boundaries: Exploring the Safe Operating Space for Humanity. Ecology and Society 14. S. $32 \mathrm{ff}$.

Umweltbundesamt (o. J.): http://www.probas.umweltbundesamt.de/php/ index.php. Aufgerufen 04.07. 2012.

\section{AUTORIN + KONTAKT}

Dr. Christiane Markard ist Fachbereichsleiterin für gesundheitlichen Umweltschutz und Schutz der Ökosysteme am Umweltbundesamt.

Dr. Christiane Markard, Umweltbundesamt, Wörlitzer Platz 1, 06844 Dessau-Roßlau. Tel.: +49 340 2103-2290, Fax:+49 340 2104-2290,

E-Mail: christiane.markard@uba.de

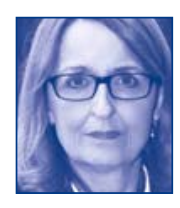

